EPJ Web of Conferences 53, 08016 (2013)

DOI: $10.1051 /$ epjconf/20135308016

(C) Owned by the authors, published by EDP Sciences, 2013

\title{
Extension of the dynamic range of large photocathode PMTs for a UHECR detector
}

\author{
M. Aglietta ${ }^{a}$, A. Castellina, S. Maldera and C. Morello \\ INAF Osservatorio Astrofisico di Torino and INFN - Sezione di Torino, \\ Corso Fiume 4, 10133 Torino, Italy
}

\begin{abstract}
Ground arrays for UHECR shower detection based on traditional counters, water Cerenkov tanks or scintillator modules, are unavoidably limited by the saturation suffered by the counters nearest to the shower axis. Reducing to a negligible level the amount of events recorded with saturated counters should be mandatory in a future UHECR ground array. The use of the signals extracted from the internal dynodes of the used photomultipliers can offer an elegant and inexpensive way to increase the dynamic range of such detectors. The viability of this technique has been explored studying in laboratory the performances of a sample of 3 Hamamatsu R5912-MOD photomultipliers. Exploiting the signal from the fifth dynode, a linear response up to an equivalent anodic peak current larger than $1 \mathrm{~A}$ (at gain $G=2 \cdot 10^{5}$ ) has been measured for all the studied PMTs. The feasibility of this technique in the frame of a new ground array for UHECR studies should be verified with a larger sample of photomultipliers.
\end{abstract}

\section{INTRODUCTION}

One of the main limitations suffered by EAS arrays (water Cherenkov or scintillation detectors) aiming at sampling the number of particles at ground level is the saturation of the counters close to the shower core, due to the high particle density hitting the detectors. The incomplete response from these detectors prevents the knowledge of the shower development near the shower core, limiting the accuracy in the reconstruction of the lateral particle distribution and increasing the uncertainty of the energy estimation. A next generation UHECR ground array should reduce the number of events with saturated detectors down to a negligible fraction. The saturation of the detectors is directly related to the dynamic range of the used photomultiplier.

Montecarlo simulations show that a $10^{21} \mathrm{eV}$ EAS generates at $200 \mathrm{~m}$ from the core a peak photocathode current of about $12.5 \mu \mathrm{A}$ in an Auger-like water Cherenkov detector. The present limit of photomultipliers like those operating at the Pierre Auger Observatory (Photonis XP1805) is 200 nA [1], at the nominal gain $G=2 \cdot 10^{5}$. The use of these PMTs in the highest energy range implies therefore an extension of the dynamic range by a factor $\sim 60$. The PMT saturation is due to the screening effect by the large number of electrons collected on the anode, that turns down the effective potential between the dynodes. This effect is largely reduced when considering the inner dynodes, where the number of electron involved is lower and a wider linear response is expected. A possible and cost-effective technique for extending the linear range of a photomultiplier is thus to extract the signals not only form the anode but also from some inner dynodes.

A complete study of the inner dynodes response has been carried out in the past by the IPN Orsay group on the Photonis XP1805 PMT [2]: they concluded that exploiting a signal output from the

\footnotetext{
ae-mail: aglietta@to.infn.it
}

This is an Open Access article distributed under the terms of the Creative Commons Attribution License 2.0, which permits unrestricted use, distribution, and reproduction in any medium, provided the original work is properly cited. 

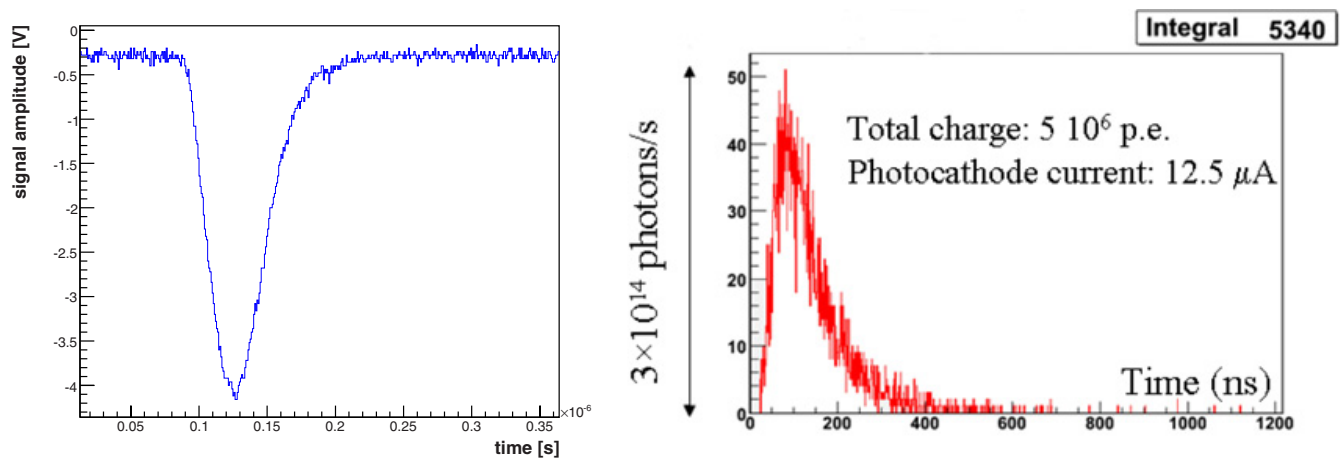

Figure 1. Left: typical mean typical response curve of a PMT anode to a LED light pulse. The mean is made over 512 samples. Right: expected photomultiplier signal in a Auger-like water Cherenkov detector, located at 250 meters from core position, for a simulated $10^{21} \mathrm{eV}$ proton shower (simulation from I.Lhenry-Yvon, IPN-Orsay).

$5^{\text {th }}$ dynode, the available linear range was at least a factor of 20 larger than the present Auger water Cherenkov detectors one. Unfortunately the study on the XP1805 photomultiplier could not be carried on because Photonis stopped the PMT production in 2009.

In this work a similar study on three Hamamatsu R5912-MOD photomultipliers (ZW-0001, ZW0019, ZW-0022) performed by the Torino group working closely together with the IPN Orsay group is reported. The signals of all the dynodes from the $8^{\text {th }}$ (D8) to the $4^{\text {th }}$ (D4) have been extracted, and their response has been compared to the anodic one assumed as reference signal.

\section{EXPERIMENTAL SET UP}

The R5912-MOD is a 8 inches PMT with 8 dynodes. It is a modified version of the standard 10 dynodes model, optimized by Hamamatsu company for improving the linearity response at low gain. The linearity performances of the R5912-MOD dynode signals have been measured generating light pulses with a pair of high intensity blue LED flashers driven by a digital controller. The PMTs were located in a dark box with the photocathode facing a diffusive volume. The light of the LED flashers is reflected by the white walls of this volume ensuring a uniform illumination.

The measurements have been performed on the signal of the $8^{\text {th }}$ dynode (D8) up to the $4^{\text {th }}$ (D4), D8 being amplified by a factor $\approx 32$ and only used for the single electron measurement and gain setting. All the studied PMTs have been operated at a gain $G \sim 2 \times 10^{5}$ (that corresponds to the nominal gain of the PMTs employed in the Auger water Cherenkov detectors). The required high voltage values have been obtained studying the single electron spectrum.

The PMT base prototype used for reading the dynode signals was provided by the IPN Orsay group. In order to prevent possible dynode voltage fluctuations due to the high impulsive current through the decoupling capacitors, individual power supplies for each dynode have been adopted. The output signals were recorded by a digital oscilloscope (2.5 Gsample/s).

The rise time of the LED light pulses, when diffused inside the white volume of the dark box, is $\sim 20 \mathrm{~ns}$ (see Fig. 1 left). This value should be compared with the $\sim 60 \mathrm{~ns}$ rise time of a simulated $10^{21} \mathrm{eV}$ event in a water Cherenkov detector presented in Fig. 1 (right): as the PMT response for slower signals behaves better, the measurement performed with this set-up should be considered conservative, particularly concerning the accuracies and the quality of the signals extracted the lowest dynodes. 

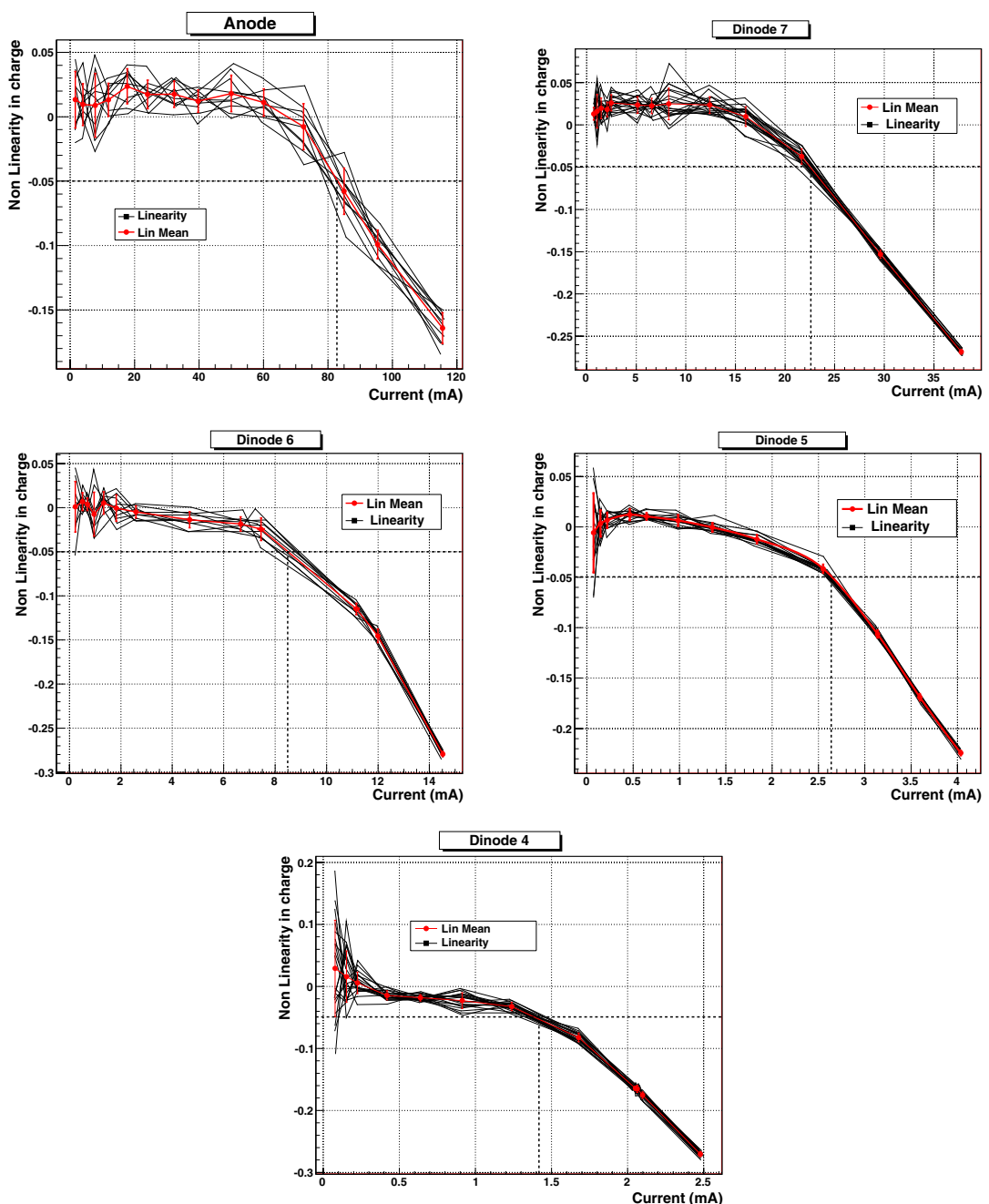

Figure 2. Measured nonlinearity as a function of peak current for the anode and the dynodes of PMT ZW-019 The horizontal dashed line represents the $-5 \%$ limit and the vertical one the corresponding peak current values. All the values are summarized in Table 1. Each black line represents a single measurement, the red one their mean value. The error bars of the mean are given by the RMS of the different measurement distribution.

\section{LINEARITY AND DYNAMIC RANGE MEASUREMENT}

The measurements have been performed by means of the "two LED technique", a method that can be easily be operated in the field for periodical PMT checks. The non-linearity $(N L)$ of the PMT output under investigation is given by the following formula:

$$
N L=\frac{\left(S_{1}+S_{2}\right)-S_{12}}{S_{1}+S_{2}}
$$

being $S_{i}$ the pulse charge for the $i$-th LED, "12" corresponding to both LEDs firing at the same time. A PMT output is considered outside of the linear region when the measured non-linearity is smaller then $-5 \%$. 


\section{EPJ Web of Conferences}

Table 1. Dynamic range for the 3 PMTs of the test bench: The $3^{r d}$ column gives the fitted values of the ratio between successive dynodes. The $4^{\text {th }}$ column represents the total ratio between a given dynode and the anode. The $5^{\text {th }}$ and the $6^{\text {th }}$ ones give the peak current value corresponding to the $-5 \%$ nonlinearity on the different dynodes and their equivalent in anode peak amplitude.

\begin{tabular}{|c|c|c|c|c|c|}
\hline \# Serial & \#Dynode & $R_{D_{i+1} / D_{i}}^{G}$ & $R_{A / D_{i}}^{G}$ & $I_{D_{i}}^{n L 5 \%}[\mathrm{~mA}]$ & $I_{D_{i} \rightarrow A}^{n L 5 \%}[\mathrm{~mA}]$ \\
\hline \multirow[t]{5}{*}{022} & $A$ & - & - & $135 \pm 1$ & 135 \\
\hline & $D 7$ & $12.1 \pm 0.1$ & $12.1 \pm 0.1$ & $31.2 \pm 0.25$ & 378 \\
\hline & D6 & $4.77 \pm 0.04$ & $57.7 \pm 0.6$ & $10.1 \pm 0.1$ & 583 \\
\hline & D5 & $6.70 \pm 0.05$ & $387 \pm 5$ & $3.42 \pm 0.03$ & 1323 \\
\hline & $D 4$ & $1.86 \pm 0.01$ & $719 \pm 10$ & $1.51 \pm 0.01$ & 1086 \\
\hline \multirow[t]{5}{*}{019} & $A$ & - & - & $83.0 \pm 0.8$ & 83 \\
\hline & $D 7$ & $11.5 \pm 0.1$ & $11.5 \pm 0.1$ & $22.8 \pm 0.2$ & 262 \\
\hline & $D 6$ & $5.6 \pm 0.2$ & $65 \pm 2$ & $8.5 \pm 0.2$ & 552 \\
\hline & D5 & $6.56 \pm 0.14$ & $422 \pm 18$ & $2.65 \pm 0.03$ & 1118 \\
\hline & $D 4$ & $1.72 \pm 0.05$ & $727 \pm 37$ & $1.45 \pm 0.01$ & 1059 \\
\hline \multirow[t]{5}{*}{001} & $A$ & - & - & $160 \pm 1$ & 160 \\
\hline & $D 7$ & $8.3 \pm 0.1$ & $8.3 \pm 0.1$ & $15.2 \pm 0.1$ & 126 \\
\hline & D6 & $5.51 \pm 0.05$ & $45.7 \pm 0.7$ & $12.2 \pm 0.1$ & 560 \\
\hline & D5 & $6.28 \pm 0.04$ & $287.2 \pm 5$ & $3.50 \pm 0.03$ & 1005 \\
\hline & $D 4$ & $1.82 \pm 0.01$ & $523 \pm 10$ & $1.51 \pm 0.02$ & 790 \\
\hline
\end{tabular}

The linearity curves for the anode and D4 to D7 dynodes of PMT ZW-019 are shown in Figure 2: the value of $N L$ as a function of the peak current for each of the PMT output is depicted. For each tube and dynode output, the signal's peak current $\left(I_{D_{i}}^{n L 5 \%}\right)$ corresponding to a non linearity of $-5 \%$ are summarized in table 1 ( $5^{\text {th }}$ column). The determination of the peak value is affected by an uncertainty of the order of $10 \%$, as the shape of the signals from the inner dynodes (mainly D4 and D5) sometimes shows oscillations probably due to the sharp rise-time of the light pulses. 


\section{UHECR 2012}
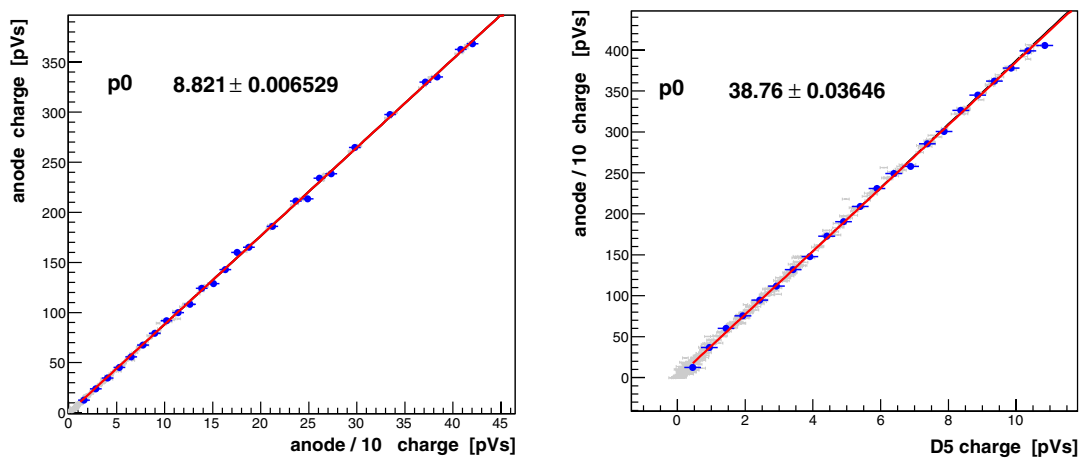

Figure 3. Direct inter-calibration between the anode and the $5^{\text {th }}$ dynode, using the prototype electronics developed for the Auger North RDA. Left: Ratio between anode and anode/10 channels. Right: Ratio between anode/10 and $5^{\text {th }}$ dynode.

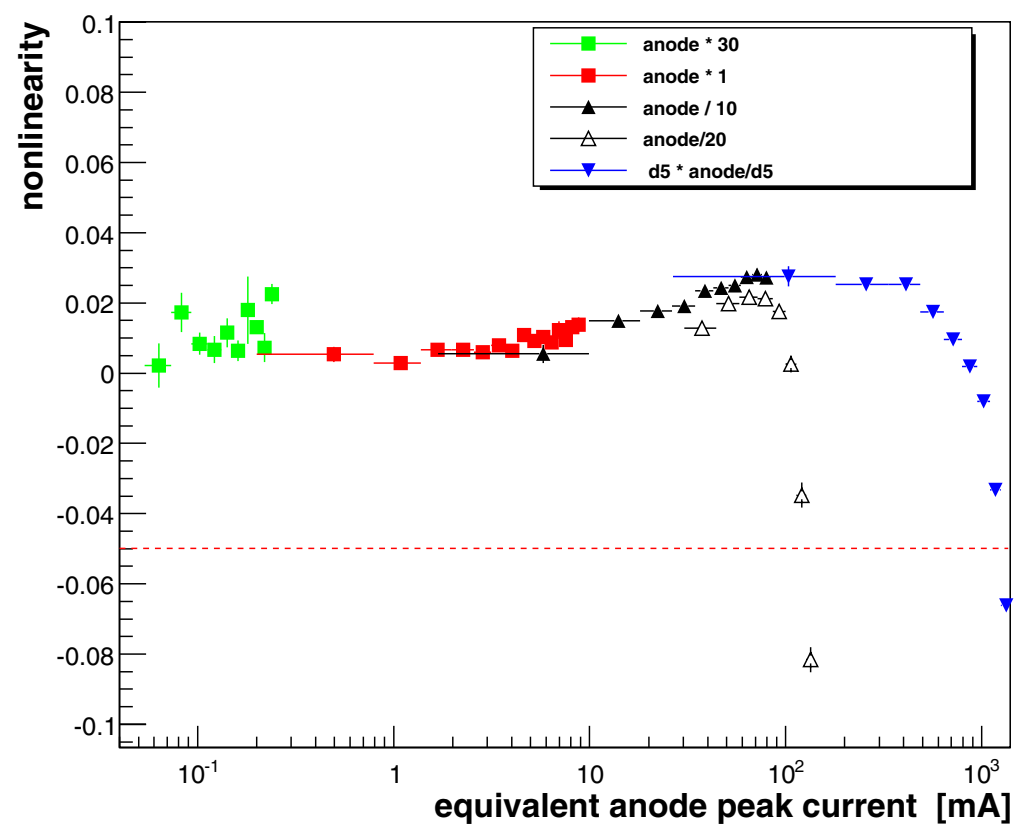

Figure 4. Non linearity as a function of equivalent anode peak current, for the anode and the $5^{\text {th }}$ dynode of PMT 022. This measurement is performed using a prototype electronics designed by APC and IPN-Orasy for the Auger North RDA. The anode signal is split over four different channels to match the fADC input range (the channel denominated anode/20 has been added to show the anode saturation current). The red dashed line represents the $-5 \%$ nonlinearity limit. The use of the signal from the $5^{\text {th }}$ dynode extends the linear range by about a factor 10 with respect to the anode. A small over-linearity (2-3\%) at large peak currents has been observed in all three studied PMTs. We notice that a similar behavior was seen also on the Photonis XP1805 PMTs (when such PMTs were studied for the Auger surface detector), and it was reduced by optimizing the geometry of the dynodes.

In order to get the effective range, the maximum peak current of each dynode needs to be converted to an equivalent anode current. The gain ratio between the anode and the different dynode outputs has been measured following a step by step procedure: using light pulses with increasing intensity, we computed the ratio in charge, $\left(R_{D_{i+1}}^{G} D_{i}\right)$ between adjacent dynodes $\mathrm{D}_{i}$ and $\mathrm{D}_{i+1}$, up to the anode, limiting the light intensity in the range where both the considered dynodes are still in their linear regime. 


\section{EPJ Web of Conferences}

The corresponding values are shown in Table 1 ( $3^{\text {th }}$ column), the obtained gain ratios between the anode and each dynode $\left(R_{A}^{G} / D_{i}\right)$ are also shown $\left(4^{\text {th }}\right.$ column).

Finally, knowing the gain ratio between the anode and all the measured dynodes we can calculate the maximum peak current for the linear regime in terms of equivalent anode current, using the following formula:

$$
I_{A \rightarrow D_{i}}^{n L 5 \%}=R_{A / D_{i}}^{G} \cdot I_{D_{i}}^{n L 5 \%}
$$

where $R_{A / D_{i}}^{G}$ is the gain ratio between the dynode $\mathrm{D}_{i}$ and the anode. The results for each PMT with the equivalent anode peak current limits, for all the dynodes measured are collected in Table 1 ( $6^{\text {th }}$ column). Despite the large differences among different PMTs, for all the tubes the best result in term of dynamic range comes from the $5^{\text {th }}$ dynode.

\subsection{Measurements with prototype electronics}

The same measurements described above have been performed using a prototype electronics developed in the frame of the Auger North RDA (Research \& Development Array) [3] at APC-Paris and IPNOrsay. The local station controller hosts 4 fADC with sampling rate of $100 \mathrm{MHz}, 3$ of them dedicated to the anode $(\mathrm{A} 1, \mathrm{~A} \times 30, \mathrm{~A} / 10)$ and one dedicated to the $5^{\text {th }}$ dynode, and it is connected to a digital LED controller through a CAN-bus. In this case the PMTs were powered using a single high voltage power supply and a "standard" resistive divider, in order to reproduce realistic working conditions.

The obtained measurements of the gain ratio between the anode and the the $5^{\text {th }}$ dynode and the nonlinearity curves are in good agreement with the results reported in Table 1. As an example, the direct inter-calibration between anode and $5^{t h}$ dynode of PMT ZW-022 is shown in Fig. 3, while Figure 4 shows the linearity measurement of the anode (split in four different channels) and the $5^{\text {th }}$ dynode of the same PMT, as a function of the equivalent anode peak current: the use of the D5 extends the linear range of about one order of magnitude.

\section{CONCLUSION}

The possibility of extending the linear dynamic range of a PMT has been investigated by using the dynode output signals. The linearity range of the inner dynodes of three Hamamatsu R5912-MOD PMT has been measured in laboratory. Using the signal extracted from the $5^{\text {th }}$ dynode the linear range of the PMT $(n L<|5 \%|)$ is extended of about one order of magnitude ${ }^{1}$ with respect to the anode, up to an equivalent anode peak current larger than 1A, for all the studied PMTs. The feasibility of this technique has to be verified with a larger sample of photomultipliers. The PMTs were also operated with the prototype station electronic unit, front-end, and LED-based control system developed in the frame of Auger North.

We would like to acknowledge the collaboration with APC-Paris and IPN-Orsay groups and our membership to the Pierre Auger Collaboration.

\section{References}

[1] T. Suomijarvi et al., Proceedings of $28^{\text {th }}$ ICRC HE (2003) 473-476

[2] B. Genolini et al., Auger technical note GAP 2009-13 (2009)

[3] F. Sarazin et al., Proceedings of $32^{\text {th }}$ ICRC HE1.4 0944 (2011)

\footnotetext{
${ }^{1}$ Corresponding to about a factor 30 with respect the the limit of the present Auger surface detectors.
} 